\title{
Into Action: Improving Access to Optimum Care for all Primary Immunodeficiency Patients
}

\author{
Francisco J. Espinosa-Rosales ${ }^{1}$ - Antonio Condino-Neto ${ }^{2}$. \\ José L. Franco ${ }^{3}$ • Ricardo U. Sorensen ${ }^{4,5}$
}

Received: 7 January 2016 / Accepted: 23 March 2016/Published online: 6 April 2016

(C) Springer Science+Business Media New York 2016

Primary Immunodeficiency (PI) is a group of over 300 inborn defects of the immune system, both in its hematopoietic as well as in its non-hematopoietic components. They can present with a broad spectrum of clinical features, either with infections (recurrent common or common lifethreatening or rare lifethreatening), autoinflammation, autoimmunity, malignancy, and or allergy. Previously classified as 'rare diseases' PI are no longer as rare as previously thought: today, it is estimated that PI affects more than six million people worldwide, with no gender, age or geographical boundaries.

Because PI can present in the form of 'common' infections, lack of awareness means that between 70 to $90 \%$ of PI sufferers remain undiagnosed, with their healthcare practitioners treating PI related infections as some other 'common infection'. Reacting to the urgent need to raise awareness on this condition, concerned organisations such as the African Society for Immunodeficiencies (ASID), the European Society for Immunodeficiencies (ESID), the Latin American Society for Immunodeficiencies (LASID), the Clinical Immunology Society (CIS) and foundations devoted to PI

Francisco J. Espinosa-Rosales

espinosa_francisco@yahoo.com.mx

1 Immunology and Allergy Unit, National Institute of Pediatrics, Mexico City, Mexico

2 Department of Immunology, Institute of Biomedical Sciences, University of São Paulo, São Paulo, SP, Brazil

3 Group of Primary Immunodeficiencies, School of Medicine, University of Antioquia, Medellín, Colombia

4 Department of Pediatrics, Louisiana State University Health Science Center, New Orleans, LA, USA

5 Faculty of Medicine, University of La Frontera, Temuco, Chile such as the Immune Deficiency Foundation (IDF) and the Jeffrey Modell Foundation (JMF) as well as patient organisations such as the International Patient Organisation for Primary Immunodeficiencies (IPOPI) have over the last two decades, implemented and supported awareness campaigns and activities all over the world towards physicians and the general public, to improve early diagnosis, appropriate treatment, and management of PI with a view to reducing morbidities and mortalities related to PI.

Since 2011, a global awareness-raising campaign -World PI Week - was established under the call to action: "Test. Diagnose. Treat." and is celebrated each April with the support of a global network of PI stakeholders (www. worldpiweek.org) to gather all groups active in the field around a common global platform, thereby strengthening the impact of their activities at local level.

As a result of these activities and with a number of lifesaving treatments having been developed over the last 60 years, the number of PI diagnosed and patients treated has grown every year.

However, despite these positive developments, it is deeply concerning that access to treatment varies dramatically across continents and even significantly across countries of the same region. For example, access to an early stem cell transplantation and gene therapy for severe types of immunodeficiencies is the rule in most countries in North America and Europe, whereas it is available only in a very small number of centers in Latin America and Africa. Is this acceptable? Likewise, while national health systems cover treatment with intravenous or subcutaneous immunoglobulin $\mathrm{G}$ ( $\mathrm{IgG})$ in most European countries, these treatments are not always reimbursed in poorer regions of the world despite extensive evidence that early diagnosis and proper treatment are life saving, life enhancing and always cost saving, and prevent unnecessary morbidity for PI patients. 
In view of these discrepancies, stakeholders united under the World PI Week banner raise their voices this year again to call on decision-makers, health authorities, healthcare funders, and professionals to put in place the relevant mechanisms to ensure that all PI patients can receive safe, efficient and appropriate treatments and optimum care throughout the world, for both their own health benefit, and the long-term benefit of the health system.

Patients with PI require ready access to antimicrobial and antifungal and prophylactic antibiotics and continuous access to immunoglobulin replacement therapies. Some patients also require hematopoietic stem cell transplantation (HSCT) or even gene therapy (GT), and emergency medicine, according to their needs and following their specialists' advice.

As a treatment listed on the WHO List of Essential Medicines for children and adults $[1,2]$ and an effective lifesaving treatment for the majority of PI patients, IgG should be made available to all PI patients worldwide. More specifically, since there is no single $\operatorname{IgG}$ product or method of administration that is suitable for all PI patients, all countries and PI centers should have access to a wide spectrum of IgG products in order to provide optimal care to patients. Funding mechanisms should be put in place to ensure availability and optimal dosing of IgG to enhance patients' quality of life, avoid further organ damage and lower treatment costs.

Further, all patients who need it, should receive HSCT or GT regardless of where they live. Early HSCT has demonstrated to cure several of the most severe forms of PI. Systematic neonatal screening for severe combined immunodeficiency and severe T-cell lymphopenia using the T-cell receptor excision circle quantification method is available in very few countries around the world but has shown to reduce dramatically the cost of an HSCT [3].

Unfortunately due to the lack of infrastructure and/or qualified personnel, access to these procedures is very difficult in less resourced settings and waiting time is often so long that many patients experience severe morbidity due to recurrent infections. The lack of a compatible donor is also a common issue. Bone marrow donor registries should thus be promoted in every country to increase the chances of performing a HSCT with the best possible match and therefore outcome.

There are several forms of severe deadly PI diseases in which GT has shown to be effective and safe and it is becoming a standard procedure, especially in cases where an identical donor is not available [4]. Unfortunately, it is currently performed only in very few highly specialized centers in high income countries. Policy makers together with health authorities should therefore invest in this technology to guarantee its availability when a HSCT is not feasible.
Beyond national policies, international collaboration mechanisms should be implemented between established reference centers and countries which lack the financial or technical requirements to provide IgG therapies, HSCT, GT or other lifesaving treatments.

In addition, health authorities in every country should promote access to early diagnosis and to quality specialized care in order to ensure appropriate therapy for all patients. Awareness programs result in more PI patients diagnosed every year, existing infrastructure is thus getting overwhelmed and many patients have to travel long distances to receive appropriate medical attention. New regional specialized centers should also be created to enable equitable geographical access to medical and nursing expertise in these conditions and more doctors specialized in this field should be trained to diagnose and give proper medical care to the growing number of PI patients. Similarly, coordinated pathways to transition pediatric patients reaching adulthood towards adult services should be established to ensure adequate planned care.

Because of their role in providing useful information to help physicians in making decisions and allowing pharmaceutical companies to ensure the supply of relevant medical products that meet the demand, patient registries should be put in place to help assessing the prevalence and incidence of PI. International patient registries should also be set up to provide information to centers in developing countries in which there are no national networks.

Last but not least, professional networks should be promoted to use peer-review specialized centers, patient registries, establish guidelines and raise standards of care and online networks must be organized to discuss difficult clinical cases and support physicians who live far away from specialized centers [5].

With early diagnosis, proper care and optimal treatment, PI is manageable and can save patients from lifelong disabilities, unnecessary vaccinations and lengthy hospital visits. It is thus vital that significant steps are taken at the local, regional and international levels to ensure that the above-mentioned mechanisms are put in place and allow PI patients to access the care they are entitled to.

Numerous studies have attempted to establish the prevalence of PI in various countries and have generated inconsistent results: a frequency of $0.38 / 100,000$ inhabitants was estimated in Mexico in 2007, 1.94/100,000 in the UK in 2011, 3.3/100,000 in Argentina in 2007, 5.38/100,000 in France in 2011, and 5.6/100,000 in Australia in 2007. Relying on data from registries, these estimates seem much lower than some based on specific population surveys recently undertaken in the United States, including a telephone survey carried out by Boyle \& Buckley reporting a prevalence of $86.3 / 100,000$ inhabitants [6-8]. 


\section{References}

1. http://www.who.int/medicines/publications/essentialmedicines/ EMLc_2015_FINAL_amended_AUG2015.pdf?ua=1 consulted on November 20, 2015.

2. http://www.who.int/selection medicines/committees/expert/20/ EML_2015_FINAL_amended_AUG2015.pdf?ua=1 consulted on November 20, 2015.

3. Clément MC, Mahlaoui N, Mignot C, Le Bihan C, Rabetrano H, Hoang L, Neven B, Moshous D, Cavazzana M, Blanche S, Fischer A, Audrain M, Durand-Zaleski I. Systematic neonatal screening for severe combined immunodeficiency and severe T-cell lymphopenia: Analysis of cost-effectiveness based on French real field data. J Allergy Clin Immunol. 2015;135:1589-93.
4. Ghosh S, Thrasher AJ, Gaspar HB. Gene therapy for monogenic disorders of the bone marrow. Br J Haematol. 2015. doi:10.1111/ bjh. 13520.

5. Principles of Care for Primary Immunodeficiencies http://journal. frontiersin.org/article/10.3389/fimmu.2014.00627/full

6. Bousfiha AA, Jeddane L, Ailal F, Benhsaien I, Mahlaoui N, Casanova JL, Abel L. Primary immunodeficiency diseases worldwide: more common than generally thought. J Clin Immunol. 2013;33(1):1-7.

7. Errante PR, Franco JL, Espinosa-Rosales FJ, Sorensen R, CondinoNeto A. Advances in primary immunodeficiency diseases in Latin America: epidemiology, research, and perspectives. Ann N Y Acad Sci. 2012;1250:62-72.

8. Boyle JM, Buckley RH. Population prevalence of diagnosed primary immunodeficiency diseases in the United States. J Clin Immunol. 2007;27(5):497-502. 